\title{
BayesVL: Rules for Versioning
}

\author{
Quan-Hoang Vuong $(1,2)$ \\ Viet-Phuong La ${ }^{(1,2)}$
}

(1) Al for Social Data Lab (AISDL), Vuong \& Associates; and,

(2) Centre for Interdisciplinary Social Research, Phenikaa University

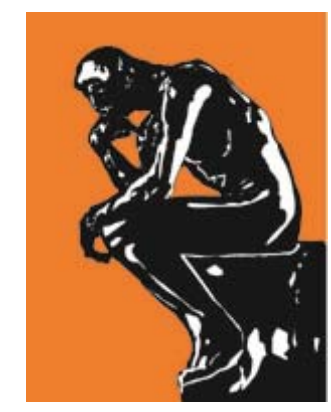

Hanoi, April 6, 2019

BayesVL is our project to develop an easy-to-use, graphics-enabled and pedagogical software package for employing Bayesian statistical analysis for nonexpert users [1].

Our rules for versioning BayesVL packages in the $\mathrm{R}$ statistical software are set for tracking the developments and progresses. In what follows [A] denotes the actual state and date of the version, and $[E]$ the estimated time.

BayesVL version $0.6[\mathrm{~A}]$ :

*Date: March 30, 2019

*Completed tasks:

- Upon the completion of BayesVL v.0.6, three modules for "estimating" basic models, namely,

a) Linear regressions for discrete and continuous variables (including interaction terms), following [2];

b) Bayesian networks, following [3]; and,

c) Nested non-linear hierarchical data models, function well in R, employing both JAGS and Stan MCMC implementations.

- Basic graphs and charts are available for use, e.g., posteriors check, distributional patterns check, robustness check.

* Limitations: Codes still need fine-tuning and calibrating efforts by the professionals facing complex modeling tasks. Modules are not integrated fully into the package, and its deployment requires technical expertise.

BayesVL version $0.7[E]$ : 
*Date: May 30, 2019

*Completed tasks:

- Adequate guidance on re-running the codes for three first sets of data, employing the models as specified in BayesVL v.0.6.

- Integration of modules into the package.

- Graphs and tests are enhanced.

- GUl for choosing desired models.

- Powering non-expert users to calibrate their chosen models and adjust parameters.

* Limitations: Codes for more complex models still need technical expertise. Tools for easy-to-use database connecting are not yet developed.

BayesVL version $0.8[\mathrm{E}]$ :

*Date: August 30, 2019

*Completed tasks:

- User-friendly tools for connecting to datasets/database, especially online publicly deposited ones, in such open repositories as Open Science Framework, Mendeley Data, or Harvard Dataverse.

- User-friendly GUls for non-expert users.

- Software packages having been tested by non-expert research workers.

* Limitations: The testing process is not complete, and feedback for further improvements/calibrations are still being collected.

BayesVL version $0.9[\mathrm{E}]$ :

*Date: October 30, 2019

*Completed tasks:

- Additional graphs and basic statistical presentations of data.

- More conventional graphs and model estimations, for comparing to those employing Bayesian modeling.

- Bayesian modeling improved with user feedback.

* Limitations: Pending technical checks and validations by programming experts.

BayesVL version $1.0[\mathrm{E}]$ :

*Date: November 30, 2019

*Completed tasks:

- Technical checks and validations by experts are done.

- Model calibrations and pre-release debugging are done.

- Real-world research applications by researchers are reported in preprints or formal publications (at least 10 publications). 
* Limitations: Pending feedbacks from wider audiences of researchers, software developers, and non-technical users.

\section{Continuous developments of BayesVL:}

The completion of BayesVL version 1.0 will mark the first draft of a research paper on BayesVL and the official release of BayesVL, including publicly open deposition of all the codes and documentation.

The package will be developing continuously, with each new version being an official release of the package.

*Note: The estimated dates of deliverables as stated above are subject to changes, when necessary, and such changes will be announced in due course.

\section{References:}

[1] Vuong QH, La VP. (2019). BayesVL package for Bayesian statistical analyses in R. Github: BayesVL version 0.6; Available from: < https://github.com/sshpa/bayesvl > (accesed: April 6, 2019).

[2] Vuong QH, La VP, Vuong TT, Nguyen VH, Ho MT, Nguyen THK, Bui QK, Ho MT. (2018). Cultural additivity: Behavioural insights from the interaction of Confucianism, Buddhism, and Taoism in folktales. Palgrave Communications, 4, 143. DOI: 10.1057/s41599-018-0189-2. URL: https://www.nature.com/articles/s41599-018-0189-2.

[3] Vuong QH, Bui QK, La VP, Vuong TT, Ho TM, Nguyen HKT, Nguyen NH, Nghiem PKC, Ho MT. (2019, January 26). Cultural evolution in Vietnam's early 20th century: a Bayesian networks analysis of Franco-Chinese house designs. arXiv Preprints, arXiv:1903.00817v1 [stat.AP]; Available from: < https://arxiv.org/abs/1903.00817 > (accessed: April 6, 2019). 